\title{
Molecular Structure of Hydrophobins Studied with Site-Directed Mutagenesis and Vibrational Sum-Frequency Generation Spectroscopy
}

\author{
K. Meister, ${ }^{*}{ }^{\dagger}$ A. Paananen, ${ }^{\dagger}$ B. Speet, ${ }^{\dagger}$ M. Lienemann, ${ }^{\ddagger}$ and H. J. Bakker ${ }^{\dagger}$ \\ ${ }^{\dagger}$ AMOLF, Science Park 104, 1098 XG Amsterdam, The Netherlands \\ ${ }^{*}$ VTT Technical Research Centre of Finland Ltd, Tietotie, FI-02150 Espoo, Finland
}

Supporting Information

ABSTRACT: Hydrophobins are surface-active fungal proteins that adsorb to the water-air interface and self-assemble into amphiphilic, water-repelling films that have a surface elasticity that is an order of magnitude higher than other molecular films. Here we use surface-specific sum-frequency generation spectroscopy (VSFG) and site-directed mutagenesis to study the properties of class I hydrophobin (HFBI) films from Trichoderma reesei at the molecular level. We identify protein specific HFBI signals in the frequency region $1200-1700 \mathrm{~cm}^{-1}$ that have not
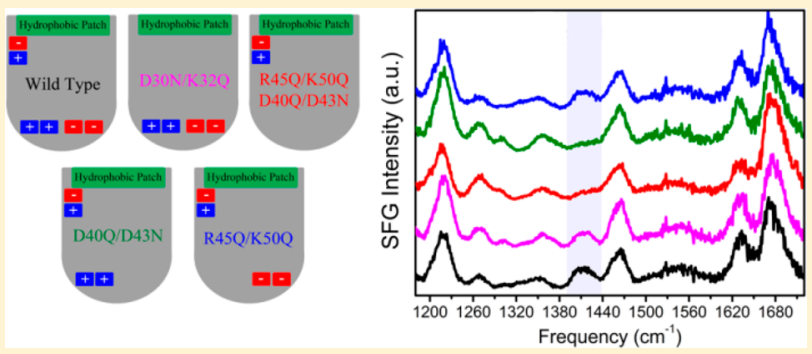
been observed in previous VSFG studies on proteins. We find evidence that the aspartic acid residue (D30) next to the hydrophobic patch is involved in lateral intermolecular protein interactions, while the two aspartic acid residues (D40, D43) opposite to the hydrophobic patch are primarily interacting with the water solvent.

\section{INTRODUCTION}

The correct functioning of many biological and chemical systems strongly depends on their interfacial structure. As such, the elucidation of the molecular-scale properties of interfacial proteins is indispensable for the development of new biomaterials and to develop effective treatments for protein related diseases that originate from conformational transitions at interfaces (Alzheimer, Parkinson). ${ }^{1}$

Hydrophobins are found exclusively in filamentous fungi and constitute an exceptional class of interfacial proteins. In nature, hydrophobins form assembled protein films that reduce the surface tension of the aqueous environment, thereby facilitating the growth of hyphae into the air and the subsequent spore release. ${ }^{2,3}$ Hydrophobin monolayers further provide a protective water repellant coating on aerial hyphae, fruiting bodies, fungal-spores, and gas cavities in lichens. ${ }^{4,5}$ The remarkable interfacial properties of hydrophobins have inspired a number of biotechnological applications, including the dispersion of otherwise insoluble drug compounds, the production of stable foams in food ingredients, and the stabilization of colloidal dispersions. ${ }^{6,7}$ In all these applications, the remarkable interfacial elasticity of hydrophobins plays a crucial role. Hence, a detailed molecular structure-function understanding of these proteins is expected to further enhance their biotechnological applicability.

Hydrophobins fold into compact and rigid structures with clearly distinguishable hydrophobic patches on their surface that render them amphiphilic, as depicted in Figure 1.,9 The fold typically consists of a four-stranded $\beta$-barrel that is stabilized by intramolecular disulfide bonds. ${ }^{8,9}$ Due to their
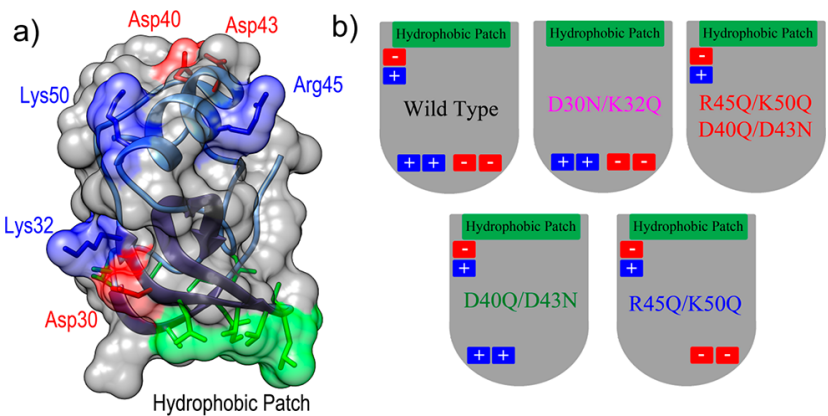

Figure 1. Three-dimensional structure of T. reesei hydrophobin HFBI (PDB: 2ZF6) and schematic structures of the investigated HFBI variants. (a) Hydrophobins fold into compact structures with a central $\beta$-barrel core and a solvent-exposed hydrophobic patch (colored in green). Basic and acidic amino acids are annotated and highlighted in blue and red, respectively. (b) Schematic structures of the HFBI variants in which particular charged residues are exchanged for neutral residues. The coloring of the variant names designates the use of colors for the data shown in Figures 2 and 3.

compactness and the clear geometric separation of their hydrophilic and hydrophobic regions are they often denoted as molecular Janus particles. ${ }^{10}$

Hydrophobins self-assemble into films thanks to their amphiphilic character. However, many of the properties of hydrophobin

Received: September 6, 2017

Revised: September 14, 2017

Published: October 2, 2017 
films cannot be explained by a simple amphiphilic model. ${ }^{11}$ Especially noteworthy is their exceptional high surface-shear modulus as observed in interfacial rheology. ${ }^{12,13}$ Hydrophobin surface assemblies are sufficiently rigid to cause a flattened plateau on top of a solution droplet. ${ }^{14}$ The analysis of the structure of hydrophobin films with AFM and diffraction techniques revealed that these films are highly ordered hexagonal structures with well-defined repeating units. ${ }^{12,15,16}$ This presence of highly regular patterns and the high interfacial elasticity of hydrophobin films suggest that hydrophobins have highly specific intermolecular and solvent interactions. ${ }^{11}$

Here we use surface-specific VSFG and site-directed mutagenesis to investigate the molecular details of class II films from T. reesei, HFBI, and four specific mutants, as schematically shown in Figure $1 \mathrm{~b}$. HFBI is a small $7.2 \mathrm{kDa}$ protein that contains six charged residues. In the variant $\mathrm{D} 30 \mathrm{~N} / \mathrm{K} 32 \mathrm{Q}$, the charged residues lysine (K32) and aspartic acid (D30) proximal to the hydrophobic patch were exchanged for electrically neutral ones. In the other three variants, the charged residues on the face opposite to the hydrophobic patch were mutated. Neutralizing the positive residues lysine (K50) and arginine (R45) leads to the negatively charged variant $\mathrm{R} 45 \mathrm{Q} / \mathrm{K} 50 \mathrm{Q}$, while neutralizing the negative aspartic acids (D40, D43) leads to the positively charged variant $\mathrm{D} 40 \mathrm{Q} / \mathrm{D} 43 \mathrm{~N}$. In the mutant $\mathrm{R} 45 \mathrm{Q} / \mathrm{K} 50 \mathrm{Q} /$ $\mathrm{D} 40 \mathrm{Q} / \mathrm{D} 43 \mathrm{~N}$, all charged residues opposite to the hydrophobic patch were exchanged for neutral residues. ${ }^{12}$ Vibrational sumfrequency generation spectroscopy (VSFG) has become a versatile surface-specific technique that can be used to probe the interfacial structure of proteins. ${ }^{17-20}$ In this technique, an infrared light pulse and a visible pulse are combined at a surface to generate light at their sum-frequency. The generation is enhanced in case the infrared light is resonant with a molecular vibration at the surface. The technique is bulk forbidden due to symmetry, and only ensembles of molecules with a net orientation, e.g., at an interface, can generate a detectable signal, thus making VSFG a highly surface-specific technique. ${ }^{21}$

\section{METHODS}

VSFG is a second-order nonlinear optical technique. It has the advantage in comparison to other classical linear optical techniques that it has an inherent interfacial selectivity for materials with inversion symmetry. Two measure VSFG signals, two laser beams, one with a fixed wavelength of $800 \mathrm{~nm}$ (VIS) and one tunable broadband infrared beam, are spatially and timely overlapped at the interface of interest. The laser source for the VSFG setup is a regenerative Ti:sapphire amplifier (Coherent) producing $800 \mathrm{~nm}$ pulses at a $1 \mathrm{kHz}$ repetition rate with a pulse duration of $35 \mathrm{fs}$ and a pulse energy of $4.5 \mathrm{~mJ}$. Approximately $85 \%$ of the laser output is used to pump a commercial highenergy optical parametric amplifier (HE-Topas, Light Conversion). This nonlinear optical device produces tunable broadband mid-IR pulses (ranging from 2 to $10 \mu \mathrm{m}, 500 \mathrm{~cm}^{-1}$ bandwidth at fwhm, 10-20 $\mu \mathrm{J})$. Another part of the $800 \mathrm{~nm}$ pulse is sent through an etalon to narrow down its bandwidth. The resulting narrow-band $800 \mathrm{~nm}$ pulse (VIS) and the broadband IR pulse are directed to the sample surface at angles of $\sim 50^{\circ}$ and $\sim 55^{\circ}$, respectively, to generate light at the sumfrequency. The VIS and IR beams are focused in spatial and temporal overlap on the sample surface with $200 \mathrm{~mm}$ and $100 \mathrm{~mm}$ focal length lenses, respectively. The SFG light generated at the surface is detected with a Princeton Instruments spectrometer system consisting of a Pixis 100 CCD camera in conjunction with an Acton SP 2356 monochromator.
The measured spectra are background subtracted (blocked IR) and normalized to a reference SFG spectrum measured from gold. As a measurement cell we used a custom-made Teflon trough. Measurements were performed in $\mathrm{H}_{2} \mathrm{O}$ (Milipore), $\mathrm{D}_{2} \mathrm{O}$ (Cambridge Isotope Labratories, 99.9\%), and in buffer solutions (100 $\mathrm{mM}$ phosphate buffer $\mathrm{pH} 7.8$, $50 \mathrm{mM}$ acetate buffer, $\mathrm{pH} \mathrm{5)}$. The protein concentration in all measurements was $13 \mu \mathrm{M}$, ensuring complete protein coverage of the water surface. ${ }^{1}$ The $\mathrm{pH}$ was adjusted by adding sodium hydroxide or hydrochloric acid. The $\mathrm{pH}$ was checked (Mettler Toledo FE20) before and after each measurement. The typical acquisition time per spectrum was $180 \mathrm{~s}$. The hydrophobins (HFBI and mutants) were purified at VTT Technical Research Centre of Finland as described previously. ${ }^{12,15}$ The purity level of the proteins is $\geq 98 \%$. The VSFG spectra of the HFBI variants in the frequency region from 1390 to $1490 \mathrm{~cm}^{-1}$ were fitted to quantify the observed changes of the intensities of the $\nu_{\mathrm{SS}, \mathrm{COO}-}\left(1420 \mathrm{~cm}^{-1}\right)$ signals. VSFG spectra were fitted with a nonresonant background and Lorentzian line shapes for the resonances as described previously. ${ }^{22}$ The results of the fits are shown in the Table S1.

\section{RESULTS}

Figure 2 shows the vibrational surface sum-frequency generation (VSFG) spectrum of aqueous solution of different

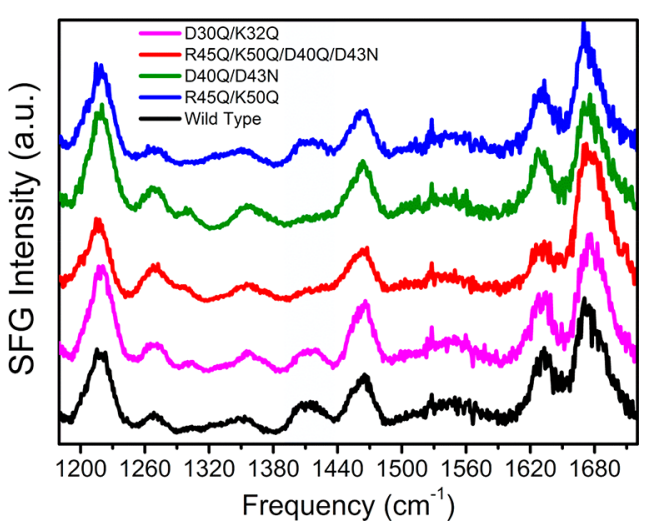

Figure 2. VSFG spectra in the frequency region from $1200-1700 \mathrm{~cm}^{-1}$ of aqueous solutions of $13 \mu \mathrm{M}$ HFBI ( $50 \mathrm{mM}$ acetate buffer, $\mathrm{pH}$ 5.0) at the solution-air surface measured with a SSP (s-SFG, s-VIS, p-IR) polarization configuration. The VSFG spectra in the frequency region from $1200-1700 \mathrm{~cm}^{-1}$ contain signals that originate from the vibrations of the protein backbone and of amino acid side chains.

variants of HFBI at the solution-air interface in the frequency region from $1200-1700 \mathrm{~cm}^{-1}$. This frequency region contains the backbone vibrations of proteins known as amide I, amide II, and amide III, and vibrational resonances originating from the side chains of the amino acids. ${ }^{23}$

In Figure 3, we present a zoom-in of the VSFG spectra of aqueous solutions $(13 \mu \mathrm{M}, 50 \mathrm{mM}$ acetate buffer, $\mathrm{pH} 5.0)$ of the different HFBI variants in the amide I region, measured with an SSP (s-SFG, s-VIS, $p$-IR) polarization configuration and with a PSP (p-SFG, s-VIS, p-IR) polarization configuration. The frequency of the amide I vibration depends on the secondary structure of the protein backbone and thus constitutes a useful tool for the conformation analysis of proteins. ${ }^{23}$ We observe strong VSFG signals at $\sim 1630$ and $\sim 1675 \mathrm{~cm}^{-1}$ for all variants in the SSP experiments. This finding indicates that all HFBI variants have similar structures at the interface. 

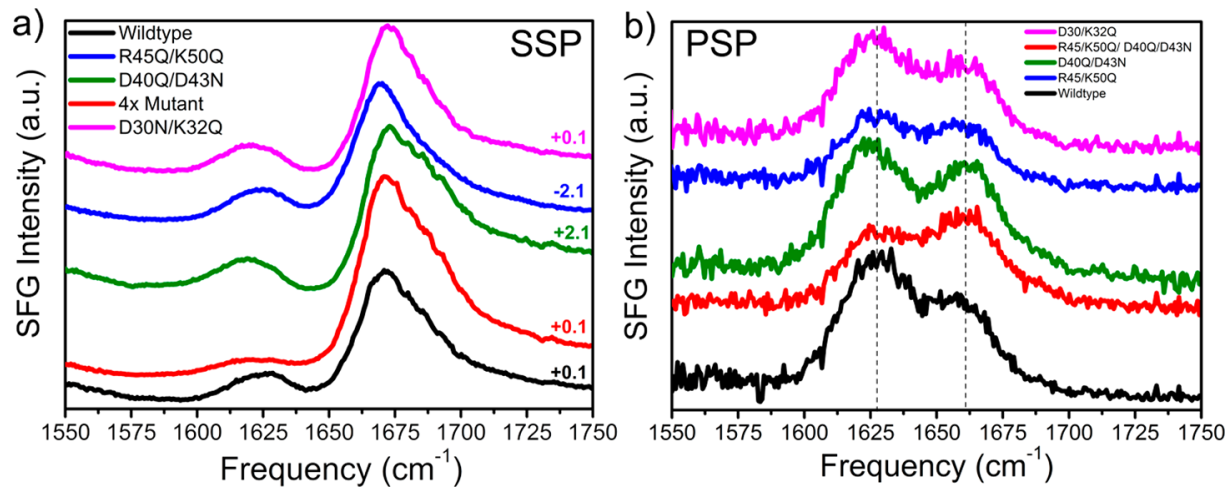

Figure 3. VSFG spectra in the frequency region $1550-1750 \mathrm{~cm}^{-1}$ of aqueous solutions of $13 \mu \mathrm{M}$ HFBI (50 mM acetate buffer, $\left.\mathrm{pH} 5.0\right)$ variants at the solution-air surface. (a) Measured with a SSP (s-SFG, s-VIS, p-IR) polarization configuration; (b) measured with a PSP polarization combination (probing the chiral vibrations). The numbers next to the VSFG spectra represent the net charge of the protein at the given experimental conditions ( $\mathrm{pH}$ 5.0).

We assign the strong signal centered at $\sim 1675 \mathrm{~cm}^{-1}$ to a combination of a $\beta$-turn signal (centered at $\sim 1665 \mathrm{~cm}^{-1}$ ) and an antiparallel $\beta$-sheet signal. ( $\mathrm{B}_{1}$-mode, centered at $\sim 1685 \mathrm{~cm}^{-1}$ ). The second amide I signal observed at $\sim 1630 \mathrm{~cm}^{-1}$ is assigned to the $\mathrm{B}_{2}$-mode of the antiparallel $\beta$-sheets. These signals originate from the central $\beta$-barrel structure, present in HFBI, and the observation and assignment of these bands agree with previous studies. $^{24,25}$

By measuring VSFG spectra with a PSP polarization configuration, we selectively probe the response of the chiral structures of the hydrophobins. ${ }^{18,26}$ As shown in Figure $3 \mathrm{~b}$, we observe chiral VSFG signals for all HFBI variants. In line with the SSP spectra, we assign the signal at $\sim 1630 \mathrm{~cm}^{-1}$ to the amide I antiparallel $\beta$-sheet $\mathrm{B}_{2}$ mode and the signal at $\sim 1660 \mathrm{~cm}^{-1}$ to the amide I vibrations of the $\beta$-turn elements present in the central $\beta$-barrel of hydrophobins. ${ }^{24}$ All HFBI variants thus show similar chiral VSFG signals, which implies that all variants remain in a folded state at the interface. For HFBI variant R45Q/K50Q/D40Q/D43N, we find that the intensity of the $\beta$-turn signal at $\sim 1675 \mathrm{~cm}^{-1}$ is much higher and the $\beta$-sheet signal at $\sim 1635 \mathrm{~cm}^{-1}$ is much lower compared to the other variants, both in the achiral spectrum of Figure 3a and in the chiral VSFG spectra shown in Figure $3 b$.

The signal at $1550 \mathrm{~cm}^{-1}$ can be assigned to the amide II vibrations of the HFBI backbone. These modes involve a combination of $\mathrm{C}-\mathrm{N}$ stretching and $\mathrm{N}-\mathrm{H}$ bending contributions. We observe no spectral differences between the mutants, which is not unexpected as amide II modes are not very sensitive to the protein conformation.

The similarity of the $1470 \mathrm{~cm}^{-1}$ band for the different variants of HFBI suggests an assignment of this band to a protein backbone mode. In case the $1470 \mathrm{~cm}^{-1}$ band would originate from amine-containing side chains (lysine, arginine, glutamine, asparagine), then differences between the mutants should be observed. The signal at $\sim 1470 \mathrm{~cm}^{-1}$ disappears upon deuteration of the protein (Figure S1). In view of these findings, we assign the band at $\sim 1470 \mathrm{~cm}^{-1}$ to the overtone of the backbone amide $\mathrm{V}$ vibration, a vibration that primarily consists of $\mathrm{C}-\mathrm{N}$ torsion and $\mathrm{N}-\mathrm{H}$ out-of-plane bending. ${ }^{27}$

The signals observed at $\sim 1415$ and $\sim 1350 \mathrm{~cm}^{-1}$ represent vibrations of amino acid side chains. ${ }^{23} \mathrm{We}$ assign the signal at $1415 \mathrm{~cm}^{-1}$ to the symmetric stretch vibration of the carboxylate anion group that results from the deprotonation of the aspartic acid residues D40 and D43. This peak assignment agrees well with previous studies. ${ }^{25,28}$ We observe clear spectral differences between the mutants signal at $\sim 1415 \mathrm{~cm}^{-1}$, as highlighted in the blue-shaded region in Figure 2 and the Figure S2. We observe a strong signal for the wild type and variants $\mathrm{D} 30 \mathrm{~N} / \mathrm{K} 32 \mathrm{Q}$ and $\mathrm{R} 45 \mathrm{Q} / \mathrm{K} 50 \mathrm{Q}$, while significantly reduced signals are found for variants $\mathrm{D} 40 \mathrm{Q} / \mathrm{D} 43 \mathrm{~N}$ and $\mathrm{R} 45 \mathrm{Q} / \mathrm{K} 50 \mathrm{Q} /$ $\mathrm{D} 40 \mathrm{Q} / \mathrm{D} 43 \mathrm{~N}$. In $\mathrm{D} 40 \mathrm{Q} / \mathrm{D} 43 \mathrm{~N}$ and $\mathrm{R} 45 \mathrm{Q} / \mathrm{K} 50 \mathrm{Q} / \mathrm{D} 40 \mathrm{Q} /$ $\mathrm{D} 43 \mathrm{~N}$, the aspartic acids $\mathrm{D} 40$ and D43 that are located opposite to the hydrophobic patch were exchanged for neutral amino acids. Interestingly, the exchange of the aspartic acid to asparagine at residue 30 proximal to the hydrophobic patch (D30N) causes no changes in the signal intensity at $1415 \mathrm{~cm}^{-1}$.

The broad signal at $\sim 1350 \mathrm{~cm}^{-1}$ can be assigned to deformation vibrations of methyl groups. The width and asymmetry of this band indicates the presence of two underlying signals and can be explained from the presence of two adjacent methyl groups in valines or leucines. ${ }^{23}$ These amino acids are vastly present in the hydrophobic patch of HFBI.

The amide III region $\left(1200-1400 \mathrm{~cm}^{-1}\right)$ is very sensitive to protein conformations. Compared to the amide I region this region has the advantages of better resolvable signals of secondary structure elements and no interference with vibrational signals from water. ${ }^{19}$ All HFBI variants show signals at $\sim 1220$ and $\sim 1270 \mathrm{~cm}^{-1}$ that we assign to the amide III band of antiparallel $\beta$-sheet and $\beta$-turn structures, respectively. We find that for the HFBI variant $\mathrm{R} 45 \mathrm{Q} / \mathrm{K} 50 \mathrm{Q} / \mathrm{D} 40 \mathrm{Q} / \mathrm{D} 43 \mathrm{~N}$ the $\beta$-sheet signal at $\sim 1220 \mathrm{~cm}^{-1}$ is weaker and the $\beta$-turn $\sim 1270 \mathrm{~cm}^{-1}$ signal is stronger compared to the other variants, which is consistent with the observations in the amide I region. The weak signal at $\sim 1300 \mathrm{~cm}^{-1}$ is assigned to the amide III band of the $\alpha$-helical segment of HFBI. ${ }^{25,29}$ The different variants show slight differences in intensity of this signal at $1300 \mathrm{~cm}^{-1}$. The changes in the intensity of the $\alpha$-helical signal at $\sim 1300 \mathrm{~cm}^{-1}$ can also explain the apparent changes in the spectral region from 1320 to $1370 \mathrm{~cm}^{-1}$. These spectral changes are unlikely due to changes in the net orientation of the $\mathrm{CH}$ groups, as it is known that the $\delta_{\mathrm{CH} 3}$ signal at $\sim 1375 \mathrm{~cm}^{-1}$ is insensitive to hydrocarbon chain conformations. Therefore, we explain the observed changes in the $1320-1370 \mathrm{~cm}^{-1}$ region from the interference of the $\mathrm{CH}$ signals with the broad amide III $\alpha$-helical signal centered at $\sim 1300 \mathrm{~cm}^{-1}$.

We can thus identify several protein specific vibrational signals in the frequency region from 1200 to $1700 \mathrm{~cm}^{-1}$ of which several have not been observed before. ${ }^{19,30}$ The assignment is summarized in Figure 4. 


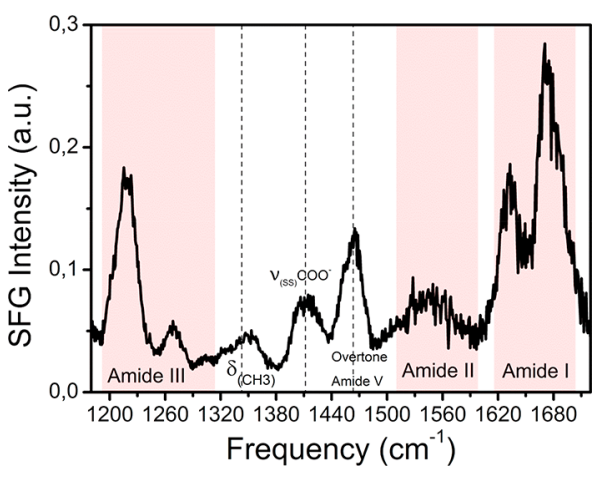

Figure 4. Vibrational surface VSFG spectra of an aqueous solution of $13 \mu \mathrm{M}$ HFBI ( $50 \mathrm{mM}$ acetate buffer, $\mathrm{pH}$ ) at the solution-air surface measured with a SSP (s-SFG, s-VIS, p-IR) polarization configuration. (a) The VSFG spectrum covers the frequency region from 1200-1700 $\mathrm{cm}^{-1}$ and consists of resonances that we assign to protein backbone (amide I-III) and amino acid side chain vibrations.

\section{DISCUSSION}

In a previous work it was found that the amide I spectrum of HFBI and HFBII depends on the $\mathrm{pH}$. This finding was explained by a modification of the intermolecular interactions and the interaction of the hydrophobin with the water solvent, following the $\mathrm{pH}$-induced change of the protonation and charge of particular amino acids. ${ }^{25}$ The removal of the acidic (D30, D40, D43) and basic amino-acids (K32, K50, R45) at the protein surface changes the net charge in the different HFBI variants (Figure S3) and is thus expected to change the interaction with the water solvent and the intermolecular interactions, thus leading to changes in the net orientation of the protein and the shape of the VSFG spectrum.

We find that the VSFG spectra of the HFBI variant $\mathrm{D} 30 \mathrm{~N} / \mathrm{K} 32 \mathrm{Q}$ look similar to the wild type spectra in both the amide I and III region which indicates that both proteins have similar conformations and orientations at the water surface, as has also been suggested by AFM experiments. ${ }^{12}$ Hence, D30 and K32 appear not to be important for the absolute interfacial orientation of the protein.

Strikingly, we do not observe a decrease of the intensity of the carboxylate band at $\sim 1415 \mathrm{~cm}^{-1}$ for the HFBI variant $\mathrm{D} 30 \mathrm{~N} / \mathrm{K} 32 \mathrm{Q}$, which indicates that residue D30 does not contribute to the VSFG intensity of this band. This observation suggests that the carboxylate anion resulting from the deprotonation of residue D30 is oriented in the plane of the surface, or that the intermolecular interactions involving the D30 residues are arranged in a well-ordered antisymmetric manner. A computational modeling study of HFBI films provided strong evidence for the presence of strong lateral intermolecular interactions in hydrophobin films, in which aspartic acid D30 and lysine K32 play a crucial role. ${ }^{16}$ Hence we conclude that residues D30 and $\mathrm{K} 32$ are not crucial for the absolute interfacial orientation, but are important for maintaining lateral intermolecular interactions. This conclusion agrees with the fact that interfacial rheology measurements showed that the loss modulus also increased most rapidly for the HFBI variant $\mathrm{D} 30 \mathrm{~N} / \mathrm{K} 32 \mathrm{Q}^{12}$

Lienemann et al. proposed that mutations opposite to the hydrophobic patch affect the protein interactions with the solvent or hydrophobin molecules in the subphase. ${ }^{12}$ In a previous study, we found evidence that in particular the interactions with the solvent play a crucial role. ${ }^{25}$ Solvation interactions with water will be strongest for carboxylate groups $\left(\mathrm{COO}^{-}\right)$, as these groups can form strong hydrogen bonds with surrounding water molecules. We observed that at high $\mathrm{pH}$, the hydrophobins acquire a more upright position, probably because such an orientation enables a better hydration of the carboxylate anion groups that result from the deprotonation of the aspartic acid residues (D30, D40, and D43). ${ }^{25}$ A change in orientation is especially noticeable in the spectral regions of the $\alpha$-helical amide vibrations, as the SFG responses of these vibrations are particularly sensitive to the protein orientation. ${ }^{24}$ Indeed, the different HFBI variants show a significant variation in the signal strength of the band at $\sim 1300 \mathrm{~cm}^{-1}$ that we assigned to the amide III vibration of $\alpha$-helical structural elements. We find that an elimination of all the charged residues opposite to the hydrophobic patch, as in R45Q/K50Q/D40Q/D43N, causes a noticeable change in the spectral VSFG response, which we explain from a different interfacial orientation of the protein, due to the altered interaction of the protein with the water solvent.

\section{CONCLUSIONS}

We recorded VSFG spectra of the protein HFBI and four variants in the frequency region from 1200 to $1700 \mathrm{~cm}^{-1}$. The mutations involve the residues lysine (K32) and aspartic acid (D30) proximal to the hydrophobic patch and the charged residues on the face opposite to the hydrophobic patch: lysine (K50), arginine (R45), and the aspartic acid residues (D40, D43). The comparison of the VSFG spectra of the different HFBI variants enables us to assign the different signal bands in the spectral region from 1200 to $1700 \mathrm{~cm}^{-1}$.

We find that the above mutations affect the strength of the interprotein interactions and their interaction with the solvent but not the overall folding state of the protein at the water surface. We find evidence that the aspartic acid residue (D30) located in the vicinity of the hydrophobic patch is involved in lateral intermolecular interactions, while the two deprotonated aspartic acids (D40, D43) located opposite to the hydrophobic patch govern the interaction with the water solvent and play an important role in the orientation of HFBI. We conclude that the combination of site-directed mutagenesis and VSFG offers an effective combination to characterize the molecular structure and intermolecular interactions of proteins at interfaces.

\section{ASSOCIATED CONTENT}

\section{S Supporting Information}

The Supporting Information is available free of charge on the ACS Publications website at DOI: 10.1021/acs.jpcb.7b08865.

Three additional figures (Figure S1-S3) and one supporting table (Table S1) (PDF)

\section{AUTHOR INFORMATION}

\section{Corresponding Author}

*E-mail: K.Meister@amolf.nl.

ORCID $\odot$

K. Meister: 0000-0002-6853-6325

Notes

The authors declare no competing financial interest.

\section{ACKNOWLEDGMENTS}

This work is financially supported by the "Nederlandse organisatie voor Wetenschappelijk Onderzoek (NWO)”. K.M. 
gratefully acknowledges the European Commission for funding through the award of a Marie Curie fellowship. A.P. and M.L. acknowledge support by the Academy of Finland through its Centres of Excellence Programme (2014-2019). Professor Markus Linder is thanked for commenting the manuscript and Riitta Suihkonen for technical assistance with hydrophobins.

\section{REFERENCES}

(1) Chiti, F.; Dobson, C. M. Protein Misfolding, Functional Amyloid, and Human Disease. Annu. Rev. Biochem. 2006, 75, 333-366.

(2) Laaksonen, P.; Szilvay, G. R.; Linder, M. B. Genetic engineering in biomimetic composites. Trends Biotechnol. 2012, 30, 191-197.

(3) Wessels, J.; De Vries, O.; Asgeirsdottir, S. A.; Schuren, F. Hydrophobin Genes Involved in Formation of Aerial Hyphae and Fruit Bodies in Schizophyllum. Plant Cell 1991, 3, 793-799.

(4) Wösten, H. A. B.; van Wetter, M.-A.; Lugones, L. G.; van der Mei, H. C.; Busscher, H. J.; Wessels, J. G. H. How a Fungus Escapes the Water to Grow into the Air. Curr. Biol. 1999, 9, 85-88.

(5) Askolin, S.; Penttilä, M.; Wösten, H. A. B.; Nakari-Setälä, T. The Trichoderma Reesei Hydrophobin Henes $\mathrm{Hfb} 1$ and $\mathrm{Hfb} 2$ Have Diverse Functions in Fungal Development. FEMS Microbiol. Lett. 2005, 253, 281-288.

(6) Cox, A. R.; Aldred, D. L.; Russell, A. B. Exceptional Stability of Food Foams Using Class II Hydrophobin HFBII. Food Hydrocolloids 2009, 23, 366-376.

(7) Wösten, H. B.; Scholtmeijer, K. Applications of Hydrophobins: Current State and Perspectives. Appl. Microbiol. Biotechnol. 2015, 99, $1587-1597$.

(8) Hakanpää, J.; Szilvay, G. R.; Kaljunen, H.; Maksimainen, M.; Linder, M.; Rouvinen, J. Two Crystal Structures of Trichoderma Reesei Hydrophobin HFBI-The Structure of a Protein Amphiphile with and without Detergent Interaction. Protein Sci. 2006, 15, 21292140.

(9) Hakanpää, J.; Paananen, A.; Askolin, S.; Nakari-Setälä, T.; Parkkinen, T.; Penttilä, M.; Linder, M. B.; Rouvinen, J. Atomic Resolution Structure of the HFBII Hydrophobin, a Self-assembling Amphiphile. J. Biol. Chem. 2004, 279, 534-539.

(10) Walther, A.; Muller, A. H. E. Janus Particles. Soft Matter 2008, 4, 663-668.

(11) Linder, M. B. Hydrophobins: Proteins that Self Assemble at Interfaces. Curr. Opin. Colloid Interface Sci. 2009, 14, 356-363.

(12) Lienemann, M.; Grunér, M. S.; Paananen, A.; Siika-aho, M.; Linder, M. B. Charge-Based Engineering of Hydrophobin HFBI: Effect on Interfacial Assembly and Interactions. Biomacromolecules 2015, 16, $1283-1292$.

(13) Cox, A. R.; Cagnol, F.; Russell, A. B.; Izzard, M. J. Surface Properties of Class II Hydrophobins from Trichoderma Reesei and Influence on Bubble Stability. Langmuir 2007, 23, 7995-8002.

(14) Yamasaki, R.; Takatsuji, Y.; Asakawa, H.; Fukuma, T.; Haruyama, T. Flattened-Top Domical Water Drops Formed through Self-Organization of Hydrophobin Membranes: A Structural and Mechanistic Study Using Atomic Force Microscopy. ACS Nano 2016, 10, 81-87.

(15) Paananen, A.; Vuorimaa, E.; Torkkeli, M.; Penttilä, M.; Kauranen, M.; Ikkala, O.; Lemmetyinen, H.; Serimaa, R.; Linder, M. B. Structural Hierarchy in Molecular Films of Two Class II Hydrophobins. Biochemistry 2003, 42, 5253-5258.

(16) Magarkar, A.; Mele, N.; Abdel-Rahman, N.; Butcher, S.; Torkkeli, M.; Serimaa, R.; Paananen, A.; Linder, M.; Bunker, A. Hydrophobin Film Structure for HFBI and HFBII and Mechanism for Accelerated Film Formation. PLoS Comput. Biol. 2014, 10, e1003745.

(17) Meister, K.; Lotze, S.; Olijve, L. L. C.; DeVries, A. L.; Duman, J. G.; Voets, I. K.; Bakker, H. J. Investigation of the Ice-Binding Site of an Insect Antifreeze Protein Using Sum-Frequency Generation Spectroscopy. J. Phys. Chem. Lett. 2015, 6, 1162-1167.

(18) Yan, E. C. Y.; Fu, L.; Wang, Z.; Liu, W. Biological Macromolecules at Interfaces Probed by Chiral Vibrational Sum
Frequency Generation Spectroscopy. Chem. Rev. 2014, 114, 84718498.

(19) Ye, S.; Li, H.; Yang, W.; Luo, Y. Accurate Determination of Interfacial Protein Secondary Structure by Combining InterfacialSensitive Amide I and Amide III Spectral Signals. J. Am. Chem. Soc. 2014, 136, 1206-1209.

(20) Devineau, S.; Inoue, K.-i.; Kusaka, R.; Urashima, S.-h.; Nihonyanagi, S.; Baigl, D.; Tsuneshige, A.; Tahara, T. Change of the Isoelectric Point of Hemoglobin at the Air/Water Interface Probed by the Orientational Flip-Flop of Water Molecules. Phys. Chem. Chem. Phys. 2017, 19, 10292-10300.

(21) Shen, Y. R. Surface Properties Probed by Second-Harmonic and Sum-Frequency Generation. Nature 1989, 337, 519-525.

(22) Strazdaite, S.; Meister, K.; Bakker, H. J. Reduced Acid Dissociation of Amino-Acids at the Surface of Water. J. Am. Chem. Soc. 2017, 139, 3716-3720.

(23) Barth, A.; Zscherp, C. What Vibrations Tell us about Proteins. Q. Rev. Biophys. 2002, 35, 369-430.

(24) Meister, K.; Bäumer, A.; Szilvay, G. R.; Paananen, A.; Bakker, H. J. Self-Assembly and Conformational Changes of Hydrophobin Classes at the Air-Water Interface. J. Phys. Chem. Lett. 2016, 7, 4067-4071.

(25) Meister, K.; Roeters, S. J.; Paananen, A.; Woutersen, S.; Versluis, J.; Szilvay, G. R.; Bakker, H. J. Observation of $\mathrm{pH}-$ Induced Protein Reorientation at the Water Surface. J. Phys. Chem. Lett. 2017, 8, 17721776.

(26) Fu, L.; Liu, J.; Yan, E. C. Y. Chiral Sum Frequency Generation Spectroscopy for Characterizing Protein Secondary Structures at Interfaces. J. Am. Chem. Soc. 2011, 133, 8094-8097.

(27) Krimm, S.; Song, S.; Asher, S. A. Amide V Overtone Assignment of a Conformation-Sensitive Band in the UV Resonance Raman Spectra of Peptides and Proteins. J. Am. Chem. Soc. 1989, 111, 42904294.

(28) Engelhardt, K.; Peukert, W.; Braunschweig, B. Vibrational SumFrequency Generation at Protein Modified Air-Water Interfaces: Effects of Molecular Structure and Surface Charging. Curr. Opin. Colloid Interface Sci. 2014, 19, 207-215.

(29) Oladepo, S. A.; Xiong, K.; Hong, Z.; Asher, S. A.; Handen, J.; Lednev, I. K. UV Resonance Raman Investigations of Peptide and Protein Structure and Dynamics. Chem. Rev. 2012, 112, 2604-2628.

(30) Ding, B.; Jasensky, J.; Li, Y.; Chen, Z. Engineering and Characterization of Peptides and Proteins at Surfaces and Interfaces: A Case Study in Surface-Sensitive Vibrational Spectroscopy. Acc. Chem. Res. 2016, 49, 1149-1157. 